\title{
In memoriam Dominique Buchillet : un engagement scientifique et politique
}

\section{Laure Emperaire}

\section{Q OpenEdition}

\section{Journals}

Édition électronique

URL : https://journals.openedition.org/jsa/17485

DOI : 10.4000/jsa. 17485

ISSN : $1957-7842$

\section{Éditeur}

Société des américanistes

\section{Édition imprimée}

Date de publication : 20 décembre 2019

Pagination : 183-190

ISSN : 0037-9174

\section{Référence électronique}

Laure Emperaire, «In memoriam Dominique Buchillet : un engagement scientifique et politique », Journal de la Société des américanistes [En ligne], 105-2 | 2019, mis en ligne le 20 décembre 2019, consulté le 04 septembre 2022. URL : http://journals.openedition.org/jsa/17485 ; DOI : https:// doi.org/10.4000/jsa. 17485 


\title{
In memoriam Dominique Buchillet: un engagement scientifique et politique
}

\author{
Laure EMPERAIRE *
}

C'est par le Brésil que la nouvelle de la disparition de Dominique, le 9 juin 2018, est arrivée. Décédée à Brest, après de nombreuses années passées au Brésil suivies de plusieurs séjours en Asie, sa retraite bretonne a été trop courte pour réaliser son nouveau projet de vie qui mêlait poursuite de ses recherches, publications et écoute de l'océan. Son dernier article paraît quelques mois avant sa disparition.

Le parcours universitaire de Dominique s'ancre à Nanterre: un Diplôme d'études universitaires générales en psychologie en 1973, une licence dans la même discipline, puis le master et le doctorat en Ethnologie. Sa trajectoire scientifique dans le haut Rio Negro, en Amazonie brésilienne, a pour point de départ son mémoire de maîtrise intitulé Analyse comparée du chamanisme dans le nord-ouest amazonien, effectué sous la direction de Patrick Menget. Son doctorat, Maladie et mémoire des origines chez les Desana du Uaupés brésilien. Conceptions de la maladie et de la thérapeutique d'une société amazonienne, est dirigé par Henri Lavondès, alors professeur au département d'ethnologie de Paris X. Il est soutenu en 1983. Ses recherches sont alors vivement appuyées par Berta Ribeiro qui travaillait à cette époque aussi dans le haut Rio Negro.

Aborder l'univers de la connaissance chamanique, soit un univers masculin, requerrait pour le moins courage et ténacité, qualités qui ne lui firent jamais défaut. Passionnée par ses recherches, elle s'y est totalement investie et a travaillé pendant plus de vingt ans dans le haut Rio Negro, en Amazonie brésilienne. Toujours attachée à comprendre les réajustements et les réinterprétations matérielles et immatérielles qui modèlent les relations interculturelles et à saisir comment les identités se redéfinissent au cours de l'histoire, ses travaux sont devenus une référence pour une jeune génération de chercheurs.

Jamais publié en tant que tel, son doctorat sera le ferment de nombreuses publications. En fin de thèse, en 1982, elle est recrutée à l'Office de la recherche scientifique et technique outre-mer (Orstom), ce qui lui permet de partir en 1984

* Directrice de recherche, IRD [laure.emperaire@ird.fr]. 
au Brésil dans le cadre de la coopération bilatérale avec le Conselho Nacional de Desenvolvimento Científico e Tecnológico (CNPq) en tant que chercheure invitée, d'abord au Museu Paraense Emílio Goeldi à Belém puis à l'Institut Socioambiental, à l'université de São Paulo, à São Paulo même et au département d'Anthropologie de l'université de Brasília. C'est à cette période que se forgent amitiés et collaborations au Brésil. Son engagement est total aux côtés des Amérindiens du haut Rio Negro, tant sur le plan scientifique que politique.

La presque totalité des grands thèmes de recherche que Dominique abordera et enrichira par la suite sont présents dans sa thèse: l'analyse des conditions de l'efficacité de la cure, la légitimité de la personne qui détient les incantations thérapeutiques et les conditions de transmission de celles-ci (Buchillet 1987a, 1990b, 1992a), les liens entre mythes et maladies (Buchillet 2013; Garnelo et Buchillet 2006a), les rapports à l'environnement (Buchillet 1988a) et, en arrièreplan, la colonisation et l'impact des missions salésiennes (Buchillet 2008). Les recherches de Dominique se situent au début d'une période clé de la vie politique brésilienne, celle de l'émergence politique du mouvement amérindien à quelques années de la fin du régime militaire en 1985 et de la promulgation en 1988 de la nouvelle constitution. Elle publie entre 1986 et 2002 de nombreuses notes pour la «Chronique du groupe d'information sur les Amérindiens » du Journal de la Société des américanistes sur des thèmes qui sont toujours, si ce n'est plus que jamais, d'actualité : la politique indigéniste au Brésil (Buchillet 1988b, 1987b, 1994a, 1995a, 1997a, 1998a, 1999a), les luttes des mouvements indigénistes pour la démarcation des terres indigènes (Buchillet 1986a, 1987c, 1993, 1996a), les visées des entreprises minières en zone indigène (Buchillet 1986b), les commémorations des « 500 ans » (Buchillet 2000a). Dominique retrace ainsi, à partir d'une analyse systématique des sources historiques, les stratégies, les politiques et les législations de la colonisation à la nouvelle constitution de 1988 qui enfin « reconnaît [aux Indiens] le droit d'être et de rester Indien » (Buchillet 1997b, p. 88; 2002a).

Sur place, au Brésil, Dominique renforce ses collaborations avec la Federação das Organizações Indígenas do Rio Negro (Fédération des organisations indigènes du Rio Negro, FOIRN) et l'Instituto Socioambiental (ISA), avec lequel elle développera plusieurs projets de coopération. Dans ce cadre, elle assure l'organisation, la révision, les notes de six des ouvrages de la série Narradores Indígenas (Umusĩ et al. 1995; Diakuru, Ksibi et Buchillet 1996, 2006; Wright 1999; Barbosa et al. 2000; W. S. Galvão, R. C. Galvão et Buchillet 2004, partenariat FOIRN-ISA). Elle poursuit en cela la démarche initiée en 1980 par Berta Ribeiro pour qui les mythes amérindiens devaient être publiés par leurs détenteurs. Elle s'investira dans la lutte pour les droits territoriaux des populations du Rio Negro, avec une expertise anthropologique pour le procureur général de la République (1990-1997), lutte qui, entre autres éléments, sera concrétisée 
en 1998 par l'homologation de la Terre indigène du haut Rio Negro à la frontière du Venezuela et de la Colombie.

Issu d'un colloque réalisé à Belém en 1989, l'ouvrage qu'elle coordonne Medicinas tradicionais e medicina ocidental na Amazônia (Buchillet [dir.] 1991) contribue à renforcer dans le monde académique et surtout parmi les organisations indigènes la visibilité de la question de la santé des populations amérindiennes et de ses interfaces, et complémentarités comme elle le défend, avec les systèmes de santé officiels (Buchillet 1991d, 1991b, p. 245). Dans ce même ouvrage, Dominique aborde les représentations indigènes sur l'origine des maladies apportées par les colonisateurs en s'attachant à analyser, dans plusieurs contextes amérindiens, comment la figure du Blanc et celle des objets manufacturés qui lui sont attachés se reconfigurent dans le système thérapeutique indigène (Buchillet 1991c, p. 167). Sa conclusion est un appel aux décideurs pour une vision élargie des conceptions de la santé indigène qui prenne en compte la diversité des contextes socio-culturels, territoriaux, écologiques et économiques des groupes amérindiens, ce qui est aussi mis en évidence par la diversité des contributions de ses collègues aux différentes parties de l'ouvrage (Dominique Gallois, Jean Langdon, Cibele Verani entre autres). Cet ouvrage contribue à divulguer au Brésil le courant de l'anthropologie médicale française selon ses propres termes (Buchillet 1991a).

Le contexte politique de cette publication est celui de la construction du nouveau système unique de santé, le SUS, issu de la Constitution de 1988. Les années 1990 sont en effet celles d'une intense réflexion partagée entre divers acteurs pour la démocratisation des services publics. La conceptualisation et l'opérationnalisation d'un système de santé indigène se traduira par la création en 1999 des Distritos sanitários especiais indígenas (DSEI) placés sous la responsabilité de la Fundação Nacional de Saúde (Fondation nationale de santé, Funasa). Dominique prendra appui sur celui du Rio Negro pour insister sur la nécessaire prise en compte du caractère processuel de la culture amérindienne et des ajustements qui en découlent entre acteurs, patients ou personnels de santé (Buchillet 2004a, p. 65).

À partir des années 1995, Dominique poursuit ses recherches sur les représentations de maladies infectieuses dites «maladies des Blancs », la variole, la rougeole, la grippe et le paludisme. Elle met en regard caractéristiques épidémiologiques de ces maladies et étiologies selon la perspective desana (Buchillet 1995b, 2002b). À cette même période, la tuberculose devient un sujet de préoccupation croissant dans le haut Rio Negro avec une incidence de cinq à dix fois supérieure à la moyenne nationale (Buchillet et Gazin 1998, en collaboration avec Pierre Gazin, épidémiologiste à l'IRD). À partir du cas de la tuberculose, c'est la question de la vulnérabilité des populations amérindiennes face à l'émergence (ou la réémergence) de nouvelles pathologies qui constituera le thème central de ses réflexions dans un contexte de forts bouleversements 
socio-culturels marqués par des contacts et migrations accrues vers la ville et des politiques de santé qui ne tiennent toujours pas suffisamment compte des contextes socio-culturels amérindiens (Buchillet 2001a). Ce pan de recherche sur les épidémies, prises aussi dans leur dimension historique, la mènera à reconsidérer l'origine multifactorielle du dépeuplement qui a suivi la colonisation, trop souvent interprété à la seule aune d'une vision biologisante des populations amérindiennes (Buchillet 2003a, 2011, 2016). Son dernier article (Buchillet 2018) retrace l'histoire des épidémies qui ont dévasté la région du Rio Negro au cours des deux derniers siècles.

Toujours préoccupée par la portée sociale de ses recherches, Dominique entreprend une colossale Bibliographie critique de la santé indigène au Brésil (3 222 références de 1840 à 2006) publiée en 2007 et disponible en ligne (Buchillet 2007). Puis, marquée par la disparition progressive de ses interlocuteurs desana, elle repart dans les années 2010 vers un projet d'anthropologie historique sur les épidémies en Asie du Sud-Est. Après une formation en médecine chinoise, elle aborde l'émergence et la diffusion des fièvres épidémiques en Thailande et en Chine du Sud sur la période 1860-1940 ainsi que les modalités de contrôle mises en œuvre. Son projet visait à retracer l'évolution des idées sur les maladies infectieuses telles qu'elles sont transcrites dans les traités de médecine traditionnelle (Buchillet 2010a, 2012, 2015; Buchillet et Lamballerie 2010b). Dominique, tel qu'elle le consigne sur la page internet où est déposée la plupart de ses publications ${ }^{1}$, avait pour ambition de mettre en regard les médecines traditionnelles amérindiennes et orientales, en particulier dans leurs interprétations des phénomènes épidémiques. Elle s'est aussi investie dans la formation accompagnant les parcours de nombre d'étudiants en master et en doctorat.

Cette guerrière, libre et généreuse, gardait aussi de multiples jardins secrets. Comme l'écrit Aloísio Cabalzar, un de ses collègues proches du Rio Negro, elle a dû rejoindre les grands kuтиa, les guérisseurs du monde qu'elle admirait tant.

Remerciements à Catherine Buchillet, Marie-José Buchillet, Alba Figueroa et Sabrina Milani pour leurs compléments et leurs relectures.

1. Pour une consultation des textes de Dominique Buchillet, voir http://horizon.documentation.ird.fr et http://www.academia.edu. 


\section{Références citées}

Barbosa Manuel Marcos, Adriano Manuel Garcia, Pedro Garcia, Benjamin Garcia et Dominique Buchillet

2000 Upiperi kalísi. Histórias de antigamente: histórias dos antigos TaliaseriPhukurana (versão do clã Kabana-idakena-yanapere), Unirva, Iauareté/ FOIRN, São Gabriel da Cachoeira, 288 p.

Buchillet Dominique

1983 Maladie et mémoire des origines chez les Desana du Uaupés (Brésil), thèse de doctorat en ethnologie, université de Paris X, Nanterre.

1986a «Droits territoriaux, raisons de sécurité nationale et politique indigéniste au Brésil », Journal de la Société des américanistes, 72, p. 230-236.

$1986 b$ «Entreprises minières et Indiens dans le haut Rio Negro », Journal de la Société des américanistes, 72, p. 236-240.

1987a «"Personne n'est là pour écouter." Les conditions de mise en forme des incantations thérapeutiques chez les Desana du Uaupés brésilien », Amerindia, 12, p. 7-32.

$1987 b$ « La politique indigéniste de la Nouvelle République », Journal de la Société des américanistes, 73, p. 263-277.

1987c «Les Indiens tukano et l'exploitation minière de leur territoire », Journal de la Société des américanistes, 72, p. 277-280.

1988a «Interpretação da doença e simbolismo ecológico entre os índios Desana », Boletim do Museu Parense Emílio Goeldi, 4 (1), p. 27-42.

1988 b «a question des droits indigènes au Brésil », Journal de la Société des américanistes, 74, p. 195-205.

1990a [1997] Os Índios da Região do Alto Rio Negro. História, Etnografia e Situação das Terras. Laudo antropológico redigido para a Procuradoria Geral da República na ação visando a demarcação sob forma única da região do alto Rio Negro (Brasilia, Brasil, junho de 1990). Revisado e ampliado em 1997, Relatório para a procuradoria Geral do Estado, Brasília, 90 p.

1990b «Los poderes del hablar. Terapia y agresión entre los índios Desana del Vaupés brasileiro », in Ellen Basso et Joel Sherzer (dir.), Las culturas nativas latinoamericanas a través de su discurso, Abya Yala, Quito/MLAL (500 Años, 24), Roma, p. 319-354.

1991a « Introdução » de « Representações e práticas das medicinas tradicionais », in Dominique Buchillet (dir.), Medicinas tradicionais e medicina ocidental na Amazônia [contribuições científicas apresentadas no Encontro de Belém - 17/novembro a 1\% dezembro de 1989], MPEG/CNPq/SCT/CEJUP/UEP, Belém, p. 63-64.

1991b « Introdução » de « A questão da integração dos sistemas médicos, problema e perspectivas », in Dominique Buchillet (dir.), Medicinas tradicionais $e$ medicina ocidental na Amazônia [contribuições científicas apresentadas no Encontro de Belém - 17/novembro a 1\% dezembro de 1989], MPEG/CNPq/ SCT/CEJUP/UEP, Belém, p. 243-246.

1991c 《Introdução » de « Impacto do contato sobre as representações tradicionais da doença e de seu tratamento ", in Dominique Buchillet (dir.), Medicinas tradicionais e medicina ocidental na Amazonia [contribuições científicas 
apresentadas no Encontro de Belém - 17/novembro a 1\% dezembro de 1989], MPEG/CNPq/SCT/CEJUP/UEP, Belém, p. 161-173.

Buchillet Dominique

1991d "A antropologia da doença e os sistemas oficiais de saúde », in Dominique Buchillet (dir.), Medicinas tradicionais e medicina ocidental na Amazônia [contribuições científicas apresentadas no Encontro de Belém - 17/novembro a 1\% dezembro de 1989], MPEG/CNPq/SCT/CEJUP/UEP, Belém, p. 21-44.

1992a «Nobody is there to hear: Desana therapeutic incantations », in E. Jean Langdon et Gerhard Baer (dir.), Portals of power: shamanism in South America, University of New Mexico Press, Albuquerque, p. 211-230.

1993 « Droits constitutionnels et démarcation des terres au Brésil », Journal de la Société des américanistes, 79, p. 225-231.

1994a «Droits indigènes, militarisation et violence contre les Indiens au Brésil », Journal de la Société des américanistes, 80, p. 243-250.

1995a «Les vieux démons de la politique indigéniste au Brésil », Journal de la Société des américanistes, 81, p. 267-273.

1995 b «Perles de verre, parures de blancs et "pots de paludisme”, épidémiologie et représentations Desana des maladies infectieuses (haut Rio Negro, Brésil) », Journal de la Société des américanistes, 81, p. 181-206.

1996a «Le décret 1775/96: une nouvelle forme de spoliation des territoires indigènes? », Journal de la Société des américanistes, 82, p. 341-347.

1997 a «Les conséquences du décret présidentiel 1775/96 pour les droits territoriaux des Indiens ", Journal de la Société des américanistes, 83, p. 295-300.

1997b « De la colonie à la République: images de l'Indien, politique et législation indigénistes au Brésil », Cahiers des Amériques latines, 23, p. 73-93.

1998a « Nouvelles menaces contre les droits des populations indiennes », Journal de la Société des américanistes, 84, p. 237-248.

1999a «Une lueur d'espoir pour les Indiens à l'aube de l'an 2000? », Journal de la Société des américanistes, 85, p. 399-409.

2000a Les « autres cinq cents ans », Journal de la Société des américanistes, 86, p. 195-214.

2001a «Tuberculose et santé publique: les multiples facteurs impliqués dans l'adhésion au traitement » Autrepart, 19, p. 71-90.

2002a «Droits constitutionnels, ressources génétiques, protection du patrimoine génétique et des savoirs traditionnels des populations indigènes », Journal de la Société des américanistes, 88, p. 245-260.

2002 b «Contas de vidro, enfeites de branco e "potes de malaria": epidemiologia e representações de doenças infecciosas entre os Desana do alto Rio Negro ", in Bruce Albert et Alcida R. Ramos (dir.), Pacificando o branco: cosmologias do contato no norte-Amazônico, IRD/UNESP, São Paulo, p. 113-142.

2003a «Contact interethnique et épidémies au Brésil », Journal international de bioéthique, 14(1-2), p. 83-99.

2004a «Cultura e saúde pública: reflexões sobre o Distrito Sanitário Especial Indígena do Rio Negro », in E. Jean Langdon et Luiza Garnelo (dir.), Saúde dos povos indígenas. Reflexões sobre antropologia participativa, ABA/Contra Capa, Rio de Janeiro, p. 53-67. 
2007 Bibliografia crítica da saúde indígena no Brasil (1844-2006), Abya Yala, Quito, http://horizon.documentation.ird.fr/exl-doc/pleins_textes/ divers14-09/010041779.pdf, consulté le 10/12/19.

2008 «Regard anthropologique sur l'œuvre décivilisatrice des missionnaires salésiens dans la région du haut rio Negro (Amazonie brésilienne) », Ateliers $d u$ Lesc, 32, http://journals.openedition.org/ateliers/2372, consulté le 10/12/19.

2010 «The 1895 epidemic outbreak of bubonic plague in Macao: a portuguese view on the transmission and spread of plague », Review of Culture, 34, p. 77-91.

2011 «Contato interétnico e saúde », in Ivo Brito (dir.), Sexualidade e saúde indigenas, IDB, Brasília, p. 43-65.

2012 «Dengue and dengue-like outbreaks in the past: the case of the Macau epidemic fever of 1874 », Infection, Genetics and Evolution, 12, p. 905-912.

2013 «Mythology, shamanism and epidemic diseases: a view from the upper Rio Negro region », in Patience Epps et Kristine Stenzel (dir.), Upper Rio Negro. Cultural and linguistic interaction in northwestern Amazonia, Museu do Índio/ Funai/Museu Nacional, Rio de Janeiro, p. 441-474.

2015 «Climate, environment and epidemic febrile diseases. A view from Chinese medicine », in Serge Morand, Jean-Pierre Dujardin, Régine Lefait-Robin et Chamnarn Apiwathnasorn (dir.), Socio-ecological dimensions of infectious diseases in Southeast Asia, Springer Singapore, Singapore, p. 9-25.

2016 «Épidémies, guerres et esclavage: les causes de la dépopulation indigène au Brésil », in Philippe Erikson (dir.), Trophées : études ethnologiques, indigénistes et amazonistes offertes à Patrick Menget, vol. 2. Guerre, chamanisme et rencontres interethniques, Société d'Ethnologie, Nanterre, p. 39-55.

2018 «Colonization and epidemic diseases in the upper Rio Negro region, Brazilian Amazon (Eighteenth-Nineteenth centuries) », Boletín de Antropología, 33 (55), p. 102-122.

Buchillet Dominique (dir.)

1991 Medicinas tradicionais e medicina ocidental na Amazônia [contribuições científicas apresentadas no Encontro de Belém - 17/novembro a 1\% dezembro de 1989], MPEG/CNPq/SCT/CEJUP/UEP, Belém.

Buchillet Dominique et Pierre Gazin

1998 «A situação da tuberculose na população indígena do alto Rio Negro (Estado do Amazonas, Brasil) », Cadernos de Saúde Pública, 14 (1), p. 181-185.

Buchillet Dominique et Xavier D. LAmballerie

2010 «The 1932 Macau epidemic of cerebrospinal meningitis: a historical perspective and critical review of the data », Infection, Genetics and Evolution, 10 , p. 896-902.

Diakuru, KisiBi (narrateurs) et Dominique Buchillet

1996 A mitologia sagrada dos antigos Desana do grupo Wari dihputiro põrã, narrateurs Américo Castro Fernandes (Diakuru) et Durvalino Moura Fernandes (Kisibi), UNIRT, Cucura do Igarapé Cucura (Amazonas, Brasil)/FOIRN (Coleção Narradores indígenas do Rio Negro, 2), São Gabriel da Cachoeira (Amazonas, Brasil)/ORSTOM, Paris, 196 p.

2006 Bueri Kãdiri Marĩriye. Os ensinamentos que não se esquecem, FOIRN (Coleção Narradores Indígenas do Rio Negro, 8), São Gabriel da Cachoeira (Amazonas, Brasil)/UNIRT, Santo Antonio (Amazonas, Brasil), 168 p. 
Galvão Wenceslau Sampaio, Raimondo Castro Galvão (narrateurs) et Dominique BuCHILlet

2004 Livro dos antigos Desana-Guahari Diputiro Porã, FOIRN (Coleção Narradores Indígenas do Rio Negro, 7)/ONIMRP, São Gabriel da Cachoeira (Amazonas, Brasil), 687 p.

Garnelo Luiza et Dominique Buchillet

2006 «Taxonomias das doenças entre os índios Baniwa (Arawak) e Desana (Tukano oriental) do alto Rio Negro (Brasil) », Horizontes Antropológicos, 12, p. 231-260.

Umusĩ Pãrõkumu, Tõrãmũ KehíRĨ et Dominique Buchillet

1995 Antes o mundo não existia: mitologia dos antigos Desana-Kehiripora, Unirt, São João Batista do Rio Tiquié/FOIRN, São Gabriel da Cachoeira.

Wright Robin M. (éd.)

1999 Waferinaipe Ianheke. A sabedoria dos nossos antepassados. Histórias dos Hohodene e dos Walipere-Dakenai do rio Aiairi, José Marcelino Cornelio, Ricardo Fontes, Manuel da Silva, Marcos da Silva, Luís Manuel, Inocêncio da Silva, María et Dominique Buchillet (narrateurs), Acira, São João Batista do Rio Tiquié/FOIRN (Coleção Narradores Indígenas do Rio Negro, 3), São Gabriel da Cachoeira (Amazonas, Brasil). 